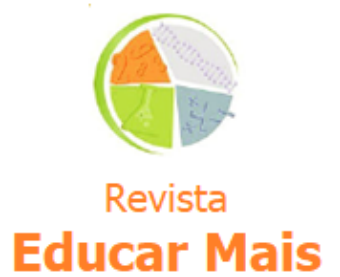

\title{
A Relação entre Conceitos Algébricos Formais e o Ensino da Álgebra nos Anos Iniciais do Ensino Fundamental
}

\section{The relationship between formal algebraic concepts and the teaching of algebra in the early years of elementary school}

Vinicius Carvalho Beckㄹ; João Alberto da Silva²

\section{RESUMO}

O objetivo deste trabalho é elucidar pontos de aproximação entre os conceitos algébricos formais e como tais conceitos são transpostos para serem trabalhados nos anos iniciais de escolaridade. Analisamos os dados a partir do referencial da teoria da transposição didática do pesquisador francês Yves Chevallard, que afirma que o conhecimento passa por três grandes etapas até ser ensinado na escola: conhecimento do sábio, conhecimento a ser ensinado e conhecimento ensinado. Esta é uma pesquisa de abordagem qualitativa, do tipo bibliográfica. Primeiramente houve uma investigação sobre a composição formal de conceitos algébricos, constituída por definições e resultados matemáticos encontrados em livros especializados de Álgebra, seguida por uma comparação e tentativa de encontrar relações com trabalhos na área da Educação que abordam especificamente o pensamento algébrico nos anos iniciais. Como conclusão geral, podemos dizer que houve forte adaptação dos conceitos algébricos formais para que fosse possível tratá-los no contexto dos anos iniciais, sobretudo no que diz respeito às representações usadas nos problemas propostos. Isto é positivo, tendo em vista que o objetivo de incluir o pensamento algébrico nos anos iniciais é desenvolver as estratégias de resolução, sem a preocupação do rigor nas representações simbólicas da criança.

Palavras-chave: Pensamento algébrico; Álgebra; Anos iniciais.

\section{ABSTRACT}

The aim of this paper is to clarify points of rapprochement between the formal algebraic concepts and how such concepts are translated to be worked in the early years of schooling. We analyze the data from the reference frame of the theory of didactic transposition the French investigator Yves Chevallard, which claims that knowledge passes through three major stages to be taught at school: knowledge of wise knowledge to be taught and knowledge taught. This is a qualitative research, bibliographic type. First there was an investigation into the formal composition of algebraic concepts consisting of definitions and mathematical results found in specialized algebra books, followed by a comparison and attempt to find relations with works in the field of education that address specifically the algebraic thinking in the initial years. As a general conclusion, we can say that there was strong adaptation of formal algebraic concepts to treat them in the context of the early years, especially with regard to the representations used in the proposed problems. This is a good thing, considering that the aim of including algebraic thinking in the early years is to develop resolution strategies, without the worry of rigour in symbolic representations of the child.

Keywords: Algebraic Thinking; Algebra; Early years.

\footnotetext{
${ }^{1}$ IFSul - Instituto Federal de Educação, Ciência e Tecnologia do Sul-rio-grandense, Pelotas/RS - Brasil.

2 FURG - Universidade Federal do Rio Grande, Rio Grande/RS - Brasil.
} 


\section{INTRODUÇÃO}

A Álgebra, enquanto área do conhecimento matemático, é utilizada há vários séculos para modelar problemas nos quais existem valores desconhecidos ou padrões matemáticos que podem ser generalizados. Podemos dizer que a fronteira do conhecimento constitui-se pelos tipos de conhecimento mais abstratos que existem.

Há alguns anos a Álgebra, que antes era vista apenas nos anos finais da educação elementar em grande parte dos países do mundo todo, passa agora a ser objeto de estudo desde os anos iniciais de escolaridade, devido a um consenso de professores e pesquisadores no que se refere a particularidade de a passagem da Aritmética para a Álgebra constituir um momento de grande dificuldade para os estudantes da educação elementar (NCTM, 2000).

O objetivo deste trabalho é elucidar pontos de aproximação entre os conceitos algébricos formais e como tais conceitos são transpostos para serem trabalhados nos anos iniciais de escolaridade.

Acreditamos que esta discussão interessa principalmente aos profissionais que trabalham nos anos iniciais do Ensino Fundamental, e que muitas vezes se deparam com conceitos algébricos, sem necessariamente estarem instrumentalizados para compreender os objetivos do trabalho com a Álgebra desde os anos iniciais.

\section{REFERENCIAL TEÓRICO}

Acreditamos ser importante apresentar as principais noções algébricas também do ponto de vista formal matemático, tendo em vista que a abordagem dos conceitos e proposições no contexto científico acabam por influenciar a escolha dos temas a serem ensinados na escola, bem como a forma como são abordados. É o que Chevallard $(1982,2005,2013)$ chama de transposição didática. Chevallard definiu mais precisamente a ideia de transposição didática em um simpósio realizado na Tchecoslováquia em 1988 (texto traduzido para a língua portuguesa por Cleonice Puggian):

A transição do conhecimento considerado como uma ferramenta a ser posto em prática, para o conhecimento como algo a ser ensinado e aprendido, é precisamente o que eu tenho chamado de transposição didática do conhecimento (CHEVALLARD, 2013, p. 9).

Segundo Chevallard (2013), existem conhecimentos que são usados como ferramenta, e outros que se destinam a ser ensinados. Também há o conhecimento a ser aprendido e ensinado em algum momento, mas que já foi conhecimento usado como ferramenta. É exatamente este processo de transformação do conhecimento, da passagem do utilizado para o ensinado, que se concentra a análise da transposição didática realizada por Chevallard. Para Chevallard (2005), o processo de transformação do conhecimento é decomposto em três etapas: conhecimento do sábio, conhecimento a ser ensinado e conhecimento ensinado.

\section{METODOLOGIA}

Esta uma pesquisa de abordagem qualitativa, do tipo bibliográfica, seguindo os critérios de May (2004). A metodologia adotada consistiu de uma primeira investigação sobre a composição formal de conceitos algébricos, constituída por definições resultados matemáticos encontrados em livros 
especializados de Álgebra, e uma posterior comparação e tentativa de encontrar relações com trabalhos na área da educação que abordam especificamente o pensamento algébrico nos anos iniciais.

\section{RESULTADOS E DISCUSSÃO}

Embora a transposição didática não componha nossa fundamentação teórica para explicar os processos de aprendizagem de conceitos algébricos no início da escolaridade, é importante observar alguns de seus argumentos a fim de compreender como os conceitos mais formais da Matemática influenciam até mesmo o currículo dos anos iniciais. Por isso, a seguir apresentamos algumas definições e resultados formais que estão relacionados com as principais noções algébricas apresentadas por Blanton et al. (2015).

\subsection{Equivalências, Expressões, Equações e Inequações}

Comecemos pelas situações de Equivalência, Expressões, Equações e Inequações. Nessas situações, o conceito de equação desempenha um papel central. Por isso, é importante definir mais precisamente o que vem a ser uma equação.

Chamamos de equação uma igualdade entre duas expressões, onde pelo menos em uma delas, mas possivelmente em ambas, figuram incógnitas $x_{1}, x_{2}, \ldots, x_{n}$, que são valores desconhecidos e que se pretende descobrir, embora nem sempre isto seja possível analiticamente (BECK, 2012, p.12).

É importante destacar na definição dada de equação a expressão valores desconhecidos, que remete a ideia de incógnita, normalmente representada por alguma letra do alfabeto latino nos livros de Matemática dos anos finais do Ensino Fundamental. Podemos dizer que esta definição formal matemática de equação concorda com a ideia de que a essência da equação está na busca por valor desconhecido.

Também é importante destacar que uma equação é uma igualdade. O conceito de igualdade também desempenha um papel central nas situações de Equivalência, Expressões, Equações e Inequações, sobretudo nos estudos sobre o pensamento relacional (CARPENTER et al., 2005; STEPHENS e WANG, 2008).

Figura 1 - Problema que envolve a Ideia de Equação

\section{Item 1a}

Fill in the blank with the value that makes the following number sentence true. How did you get your answer?

$7+3=\ldots+-4 \quad$ Why?

Fonte: Blanton et al. (2015).

Com base na definição de Beck (2012) para equação, pode-se também definir inequação: uma inequação é uma desigualdade entre duas expressões, onde pelo menos em uma delas, mas possivelmente em ambas, figuram incógnitas $x_{1}, x_{2}, \ldots, x_{n}$, que são valores desconhecidos $\mathrm{e}$ pertencentes a intervalos numéricos que se pretende estabelecer, embora nem sempre isto seja possível analiticamente. 


\subsection{Aritmética Generalizada}

As situações de Aritmética Generalizada são referentes às propriedades aritméticas das operações numéricas, assunto extremamente importante entre os matemáticos, especialmente aqueles que se dedicam ao estudo da Álgebra Abstrata, ramo da Matemática que se ocupa em analisar as estruturas algébricas e as operações que são definidas nos diferentes tipos de conjuntos em Matemática. No livro de Hefez (2014) as estruturas de conjuntos, grupo, anel e corpo são estudadas com profundidade. Normalmente esse estudo faz parte do currículo de cursos superiores de Matemática. Com relação aos assuntos tratados neste trabalho, interessa aprofundar uma pouco a discussão sobre conjuntos numéricos, e as principais operações aritméticas conhecidas, bem como suas propriedades.

A teoria básica de conjuntos pode ser encontrada em Miranda e Pena (2006). A seguir apresentamos apenas os conjuntos numéricos, importantes para o entendimento dos detalhes que permeiam as operações entre números naturais que são tratadas no presente trabalho.

Os números naturais constituem um poderoso modelo de contagem de objetos (LIMA, CARVALHO, WAGNER, MORGADO, 2006). O conjunto formado por tais números é comumente representado na literatura matemática pelo símbolo $\mathbb{N}=\{0,1,2,3 \ldots\}$. O número zero está incluído neste conjunto, significando ausência de quantidade. O conjunto dos números naturais, retirando-se o zero é denotado por $\mathbb{N}^{*}=\{1,2,3 \ldots\}$.

Ressaltamos que várias teorias matemáticas desconsideram o zero como número natural. $\mathrm{A}$ inclusão ou não do zero no conjunto dos naturais é uma convenção adotada de acordo com os objetivos de cada teoria. Como neste trabalho estamos interessados particularmente nas operações elementares, isto é, operações que envolvem apenas números naturais, precisamos lembrar que na subtração é interessante podermos subtrair duas quantidades idênticas, daí segue a escolha pela inclusão do zero.

O conjunto dos números inteiros, representado por $\mathbb{Z}=\{\ldots,-3,-2,-1,0,+1,+2,+3, \ldots\}$, também está associado com a contagem de objetos. No entanto, nos números inteiros existe a noção de sentido, ou seja, podemos contar uma quantidade positiva ou uma quantidade negativa, sendo que o significado destas expressões é convencionado de acordo com a grandeza envolvida (VERGNAUD, 1985).

As operações aritméticas elementares estudadas no início da escolaridade não envolvem números racionais. Ainda assim, o entendimento desses números é importante para a formalização da operação de divisão.

O conjunto dos números racionais é denotado por $\mathbb{Q}=\left\{\frac{m}{n} \mid m \in \mathbb{Z}, n \in \mathbb{N}^{*}\right\}$, e compreende todas as frações. Este conjunto é uma importante ferramenta para a contagem de porções, sendo uma primeira tentativa de medição fracionada, tanto no âmbito da história da humanidade, quanto na construção dos primeiros conceitos geométricos pela criança, embora as representações, nesse último caso, sejam pouco arbitrárias, e mais influenciadas pela transmissão social.

O entendimento dos números racionais é importante no estudo das inconsistências da operação de divisão, pois deve-se sempre ter em vista que o resultado desta operação não poderá extrapolar o conjunto dos números naturais nos anos iniciais de escolaridade. 
No âmbito das operações elementares, o conjunto dos racionais é o conjunto de maior abrangência, visto que todas as operações entre números naturais podem no máximo, produzir números racionais como resultado. A saber: a adição de números naturais produz apenas números naturais; a subtração de números naturais pode resultar em números inteiros; a multiplicação de números naturais resulta apenas em números naturais; e a divisão de números naturais pode resultar, no máximo, em um número racional.

Figura 2 - Problema que envolve a Ideia de Comutatividade

\section{Item 2b}

Circle True or False and explain your choice.

$$
57+22=58+21 \quad \text { True False How do you know? }
$$

Fonte: Blanton et al. (2015).

Ao longo da história, os números naturais foram formalmente abordados de várias formas. Atualmente, a fundamentação teórica aritmética baseia-se nos Axiomas de Peano (HEFEZ, 2005), a partir dos quais podem ser formalizadas as noções de antecessor, sucessor, adição, subtração, multiplicação, divisão e ordem nos números naturais.

É importante ressaltar que embora os aspectos formais da teoria dos números naturais sejam inadequados para utilização direta em sala de aula, o educador precisa ter a teoria em vista para que os conceitos sejam aprendidos pelos alunos de forma cientificamente consistente e didaticamente atualizada, isto é, conceitos constituídos de coerência matemática e em concordância com a bibliografia atual sobre o assunto.

A seguir, será descrita brevemente a teoria elementar dos números naturais. Maiores detalhes técnicos, bem como exemplos, poderão ser encontrados em: Domingues (1991); Domingues e Iezzi (2003); Hefez (2005); e também em Lima et al. (2006).

O símbolo $\mathbb{N}$ representa o conjunto dos números naturais. O que caracteriza tal conjunto é a noção de sucessor. Dados $n, n^{\prime} \in \mathbb{N}$, diz-se que $n^{\prime}$ é sucessor de $n$ quando $n^{\prime}$ representa uma quantidade maior do que $n$ e não há números naturais entre eles.

A partir da noção de sucessor, pode-se caracterizar mais precisamente o conjunto dos naturais como o conjunto que satisfaz os seguintes axiomas, conhecidos como Axiomas de Peano:

A1) Todo número natural tem um único sucessor;

A2) Números naturais diferentes possuem sucessores diferentes;

A3) Existe um único número que não é sucessor de nenhum outro. Este número é chamado de um - na língua portuguesa - e representado pelo símbolo "1";

A4) Seja $X \subset \mathbb{N}$. Se $1 \in X$, e se o sucessor de qualquer elemento de $X$ ainda está em $X$, então $X=\mathbb{N}$. (Axioma da Indução)

O restante dos números naturais é representado com o auxílio do sistema de numeração hinduarábico. Uma alternativa para representação intuitiva dos números naturais é dada pela forma extensiva do conjunto $\mathbb{N}=\{0,1,2,3,4,5, \ldots\}$. 
A seguir vamos apresentar em termos formais as operações de adição, subtração, multiplicação e divisão definidas no domínio do conjunto dos números naturais.

Chama-se adição a operação que associa dois números $n, p \in \mathbb{N}$, a um terceiro número natural, chamado de soma de $n$ e $p$, o qual é representado pelo símbolo $n+p$. A soma $n+p$ é o número natural que se obtém a partir de $n$ aplicando-se $p$ vezes seguidas a operação de tomar um sucessor. Neste caso, os números $n$ e $p$ são chamados de parcelas (LIMA et al., 2006).

Chama-se subtração a operação que associa dois números $n, p \in \mathbb{N}$, a um terceiro número natural, chamado de diferença entre $n$ e $p$, sendo representado pelo símbolo $n-p$. A diferença $n-p$, com $n>p$, é o número natural $d$ tal que $n=p+d$, para algum $d \in \mathbb{N}$. Neste caso, o número $n$ é chamado minuendo, e o número $p$ é chamado subtraendo.

Chama-se multiplicação o operação que associa dois números $n, p \in \mathbb{N}$, a um terceiro número natural, chamado de produto de $n$ e $p$, o qual é representado pelo símbolo $n \times p$. O produto $n \times p$ é o número natural que se obtém a partir da soma de $n$ parcelas de $p$. Neste caso, os números $n$ e $p$ são chamados de fatores.

Chama-se divisão a operação que associa dois números $n, p \in \mathbb{N}$, a um terceiro número natural, chamado de quociente de $n$ sobre $p$, sendo representado pelo símbolo $n \div p$. O quociente $n \div p$ é o número natural $q$ tal que $n=p \times q+r$, para alguns $q, r \in \mathbb{N}$. Neste caso, o número $n$ é chamado dividendo, o número $p$ é chamado divisore o número $r$ é chamado resto.

Chama-se comutatividade a propriedade que afirma a equivalência de resultados quando dois objetos de um conjunto são operados. Denotando pelo asterisco * uma operação qualquer, pode-se expressar em símbolos: $a * b=b * a$. As operações de adição e multiplicação são comutativas em $\mathbb{N}$. As operações de subtração e divisão não são comutativas em $\mathbb{N}$.

Chama-se associatividade a propriedade que afirma a equivalência de resultados quando três ou mais objetos são operados segundo pela mesma operação. Em símbolos: $(a * b) * c=a *(b * c)$. As operações de adição e multiplicação são associativas em $\mathbb{N}$. As operações de subtração e divisão não são.

No conjunto dos números naturais também vale a distributividade da multiplicação em relação à adição: $a \times(b+c)=a \times b+a \times c$. Como não vale para $\mathbb{N}$ a distributividade da adição em relação à multiplicação, podemos falar apenas em distributividade, sem mencionar a orientação desta propriedade em termos de operações entre números naturais.

\subsection{Pensamento Funcional: Recursividade e Padrão}

As situações de Pensamento Funcional, pela própria definição, envolvem a ideia matemática de função. Por isso optamos por aprofundar a descrição de conceitos relacionados com funções, no contexto da Matemática formal. 
Figura 3 - Problema que envolve a Ideia de Recursividade

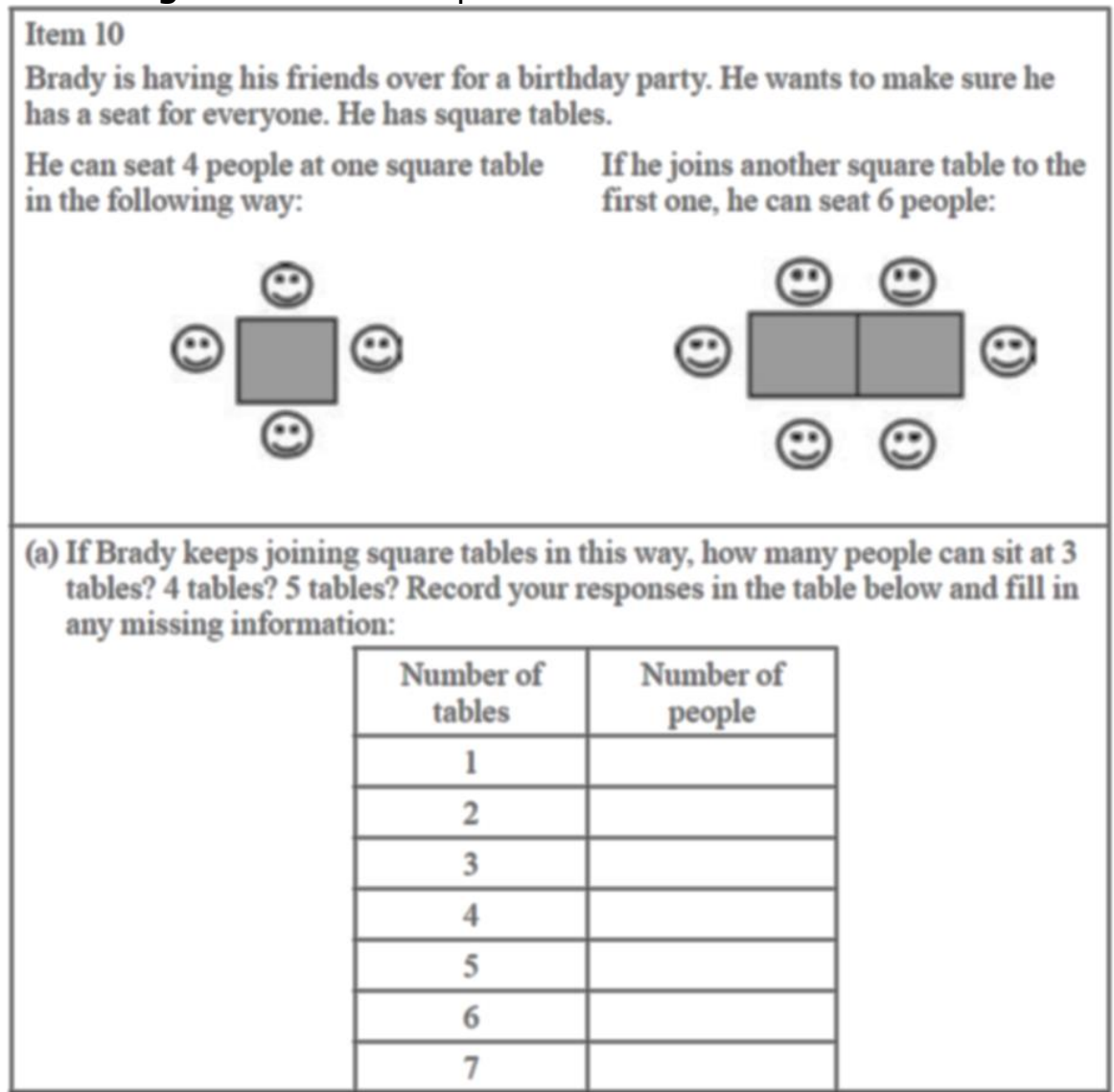

Fonte: Blanton et al. (2015).

A seguir é dada a definição técnica de função. No entanto, algumas definições preliminares são fornecidas inicialmente, como o produto cartesiano e a relação entre conjuntos. Tais noções preliminares são fornecidas a fim de definir o conceito de função nos termos da teoria clássica de conjuntos.

Dados dois conjuntos $A$ e $B$ não vazios, definimos o produto cartesiano entre $A$ e $B$, denotado por $A \times B$, como o conjunto de todos os pares ordenados da forma $(a, b)$, nos quais $a \in A$ e $b \in B$, ou equivalentemente $A \times B=\{(a, b): a \in A, b \in B\}$.

Dados dois conjuntos $A$ e $B$ não vazios. Chamamos de relação entre $A$ e $B$, qualquer subconjunto do produto cartesiano $A \times B$. Uma relação $R$ de $A$ em $B$, isto é, na qual os elementos da primeira coordenada pertencem a $A$ e os elementos da segunda coordenada pertencem a $B$, pode ser denotada por $R: A \rightarrow B$. Neste caso, o conjunto $A$ é chamado de domínio da relação e o conjunto $B$ é chamado de contradomínio da relação.

Dados dois conjuntos $A$ e $B$ não vazios. Chamamos de função toda relação que associa a cada $x \in A$, um único $y \in B$. Uma função $f$ de $A$ em $B$ pode ser denotada por $f: A \rightarrow B$. Neste caso, o conjunto $A$ é chamado de domínio da função, o conjunto $B$ é chamado de contradomínio da função e o conjunto $Y=\{y \in B: y=f(x)$, para algum $x \in A\}$ é chamado de imagem de $f$.

Alternativamente, podemos dizer que uma função é uma regra de dependência entre dois conjuntos. Uma função $f: A \rightarrow B$ é uma regra na qual os elementos de $B$ dependem dos elementos de $A$. Se $x \in$ $A$, então se representa $f(x)$ o valor do conjunto $B$ dependente de $x$. Chamamos de atribuição a substituição de $x$ por algum elemento no domínio da função $f$. 


\subsection{Ideia de Variável}

As situações envolvendo a ideia de Variável estão relacionadas com a ideia algébrica de variável, que por sua vez, está intimamente ligada à ideais de fórmula. A seguir, na figura 4, apresentamos a continuação do problema da figura 3 , porém agora com foco na regra geral que associa o número de mesas e o número de lugares.

Figura 4 - Problema que envolve a Ideia de Padrão Algébrico

(b) Do you see any patterns in the table? Describe them.

(c) Find a rule that describes the relationship between the number of tables and the number of people who can sit at the tables. Describe your rule in words.

(d) Describe your relationship using variables. What do your variables represent?

Fonte: Blanton et al. (2015).

O pensamento funcional envolve principalmente as noções de recursividade e padrões em sequências, que podem ser numéricas ou não-numéricas. A ideia de padrão algébrico está ligada principalmente com a capacidade de transformar a recursividade de um processo em uma regra geral, sendo uma das habilidades mais sofisticadas de pensamento algébrico.

Chamamos de fórmula uma representação simbólica que indica uma relação entre duas quantidades. As quantidades envolvidas em uma fórmula são chamadas de variáveis. Sendo assim, podemos dizer que a fórmula é a representação simbólica de qualquer subconjunto de um produto cartesiano, pois como vimos acima, cada subconjunto de um produto cartesiano pode ser definido como relação.

\subsection{Raciocínio Proporcional}

As situações de Raciocínio Proporcional não requerem um esclarecimento formal matemático além da própria definição da operação de multiplicação dada nesta seção, embora haja uma complexidade que transcende a ideia de que o produto é meramente o resultado dos fatores, já que no Raciocínio Proporcional os fatores podem não estar diretamente explícitos nos problemas, necessitando uma adaptação antes da realização da operação de multiplicação.

Figura 5 - Problema que envolve a Ideia de Proporcionalidade Algébrica

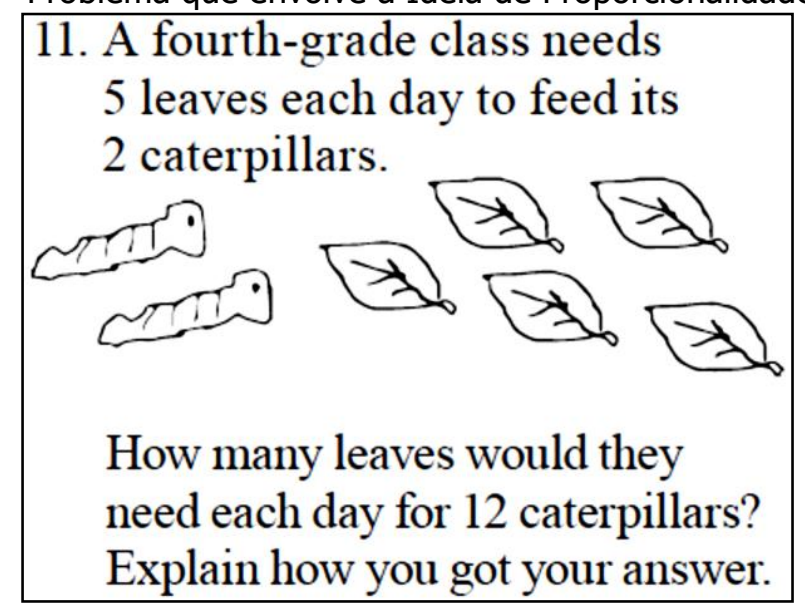

Fonte: Blanton et al. (2015). 
A ideia de Raciocínio Proporcional se aproxima bastante de situações que envolvem a noção de razão e proporção, ganhando espaço no currículo dos anos iniciais com as novas abordagens de pensamento algébrico que surgem na literatura.

\section{CONSIDERAÇÕES FINAIS}

Podemos dizer que os conceitos algébricos sofreram fortes adaptações do contexto formal algébrico, que constitui aqui o conhecimento do sábio, para o conhecimento a ser ensinado, que é aquele que aparece nos documentos oficiais e são propostos como avaliação. Neste trabalho, consideramos como uma ilustração do conhecimento a ser ensinado os problemas propostos por Blanton et al. (2015), para fins de análise.

Neste trabalho não abordamos como se estrutura o conhecimento algébrico ensinado nos anos iniciais, pois nossa preocupação foi especificamente com a adaptação do conhecimento algébrico em diferentes formatos. Sugerimos a análise do conhecimento algébrico ensinado para trabalhos futuros, assim como novas abordagens que analisam o pensamento algébrico no currículo oficial, tendo em vista que tal conhecimento ainda é novidade para grande parte da comunidade acadêmica e do sistema educacional brasileiro.

Consideramos que o objetivo do trabalho, que foi "elucidar pontos de aproximação ente os conceitos algébricos formais e como tais conceitos são transpostos para serem trabalhados nos anos iniciais de escolaridade", foi cumprido, pois foi possível estabelecer várias conexões do conhecimento algébrico formal com as novas abordagens de pensamento algébrico para anos iniciais, permitindo maior compreensão do processo de transposição didática de conceitos algébricos para os anos iniciais.

Como conclusão geral, podemos dizer que houve forte adaptação dos conceitos algébricos formais para que fosse possível tratá-los no contexto dos anos iniciais, sobretudo no que diz respeito às representações usadas nos problemas propostos. Isto é positivo, tendo em vista que o objetivo incluir o pensamento algébrico nos anos iniciais é desenvolver as estratégias de resolução, sem a preocupação do rigor nas representações simbólicas da criança.

\section{REFERÊNCIAS}

BECK, Vinicius Carvalho. Elementos de Álgebra Linear e Geometria Analítica. Editora Universidade Federal de Pelotas, Pelotas, 2012.

BLANTON, Maria; STEPHENS, Ana; KNUTH, Eric; GARDINER, Angela Murphy; ISLER, Isil; KIM, JeeSeon. The Development of Children's Algebraic Thinking: The Impact of a Comprehensive Early Algebra Intervention in Third Grade. Journal for Research in Mathematics Education, v.46, n.1, p.39-87, 2015.

CARPENTER, T. P.; LEVI, L.; FRANKE, M. L. ZERINGUE, J. K. Algebra in the elementary school: developing relational thinking. ZDM - The International Journal on Mathematics Education, v.37, n.1, p.53-59, 2005.

CHEVALLARD, Yves; JOHSUA, Marie-Alberte. Un exempled'analyse de la transposition didactique: La notion de distance. Recherches em didactique des mathématiques, v.3, n.2, 1982. 
CHEVALLARD, Yves. La transposiciónDidática: del saber sabio al saber enseñado. Buenos Aires: Aique Grupo Editor, 2005.

CHEVALLARD, Yves. Sobre a teoria da transposiçãodidática: algumasconsideraçõesintrodutórias. Revista de Educação, Ciências e Matemática, v.3, n.2, 2013.

DOMINGUES, Hygino H. Fundamentos de aritmética. Editora Atual, São Paulo, 1991.

DOMINGUES, Hygino H.; IEZZI, Gelson. Álgebra moderna. Editora Atual, 4. ed., São Paulo, 2003. HEFEZ, Abramo. Elementos de aritmética. Sociedade Brasileira de Matemática, Rio de Janeiro, 2005.

HEFEZ, Abramo. Curso de Álgebra, volume 1. 5 ed. Sociedade Brasileira de Matemática, Rio de Janeiro, 2014.

LIMA, Elon Lages; CARVALHO, Paulo Cezar Pinto; WAGNER, Eduardo; MORGADO, Augusto César. A Matemática do Ensino Médio: volume 1. Sociedade Brasileira de Matemática, Rio de Janeiro, 2006.

MAY, Tim. Pesquisa social: questões, métodos e processo. Porto Alegre, Artmed, 2004.

MIRANDA, Maria Virginia; PENA, Fernando Souza da. Teoria dos Conjuntos. Instituto Piaget, 2006. 268p.

NCTM. 2000. Princípios e Normas para a Matemática Escolar. (1.ed. 2000) Tradução portuguesa dos Principles and Standards for School Mathematics. 2.ed., APM, Lisboa, 2008.

STEPHENS, M.; WANG, X. Investigating some junctures in relational thinking: a study of year 6 and 7 students from Australia and China. Journal of Mathematics Education, v.1, n.1, p.28-39, 2008.

VERGNAUD, Gérard. 1985. A criança, a matemática e a realidade: problemas do ensino da matemática na escola elementar. Tradução de Maria Lucia Faria Moro. 3.ed. Curitiba: Editora da UFPR, 2009. 